\title{
Global and local properties of finite groups with only finitely many central units in their integral group ring
}

\author{
Andreas Bächle, Mauricio Caicedo, Eric Jespers* and \\ Sugandha Maheshwary
}

Communicated by Britta Späth

\begin{abstract}
The aim of this article is to explore global and local properties of finite groups whose integral group rings have only trivial central units, so-called cut groups. For such a group, we study actions of Galois groups on its character table and show that the natural actions on the rows and columns are essentially the same; in particular, the number of rational-valued irreducible characters coincides with the number of rational-valued conjugacy classes. Further, we prove a natural criterion for nilpotent groups of class 2 to be cut and give a complete list of simple cut groups. Also, the impact of the cut property on Sylow 3 -subgroups is discussed. We also collect substantial data on groups which indicates that the class of cut groups is surprisingly large. Several open problems are included.
\end{abstract}

\section{Introduction}

Let $G$ be a finite group, and let $\mathcal{U}(\mathbb{Z} G)$ denote the group of units of its integral group ring $\mathbb{Z} G$. The most prominent elements of $\mathcal{U}(\mathbb{Z} G)$ are surely $\pm G$, the trivial units. In the case these are all the units, this gives tight control on the group $G$, and all the groups with this property were explicitly described by G. Higman [22]. If the condition is only put on the central elements, that is, $\mathbb{Z}(\mathcal{U}(\mathbb{Z} G))$, the center of the units of $\mathbb{Z} G$, only consists of the "obvious" elements, namely $\pm \mathcal{Z}(G)$, then the situation is vastly less restrictive, and these groups are far from being completely understood. We will however show that this condition is restrictive enough to reveal many of their interesting properties. These groups $G$ with $\mathbb{Z}(\mathcal{U}(\mathbb{Z} G))= \pm \mathbb{Z}(G)$, i.e. all central units of $\mathbb{Z} G$ are trivial, are called cut groups, a name coined in [8]. The question of classifying cut groups is also included in the collection of major open group ring problems in S. K. Sehgal's path-breaking book [41, Problem 26].

The work of the first and the second author was supported by postdoctoral fellowships of the FWO (Research Foundation Flanders). The third author is supported in part by Onderzoeksraad of Vrije Universiteit Brussel and FWO (Research Foundation Flanders). The research of the fourth author is supported by Department of Science and Technology (DST), India (INSPIRE/04/2017/000897). 
The study of cut group dates back at least to the 1970s, and some cornerstones were a chapter in a book of A. A. Bovdi [12] and an article by J. Ritter and Sehgal [38]. There, the condition for being a cut group was reformulated in a group theoretic condition on $G$, also termed the RS-property (see [9]). An immediate consequence is that rational groups (i.e. groups whose character tables only contain rational entries), e.g. symmetric groups $S_{n}$ and Weyl groups of complex Lie algebras, are cut groups. The class of rational groups is of vital interest in the representation theory of finite groups and gives a nice connection to this area. In [14], D. Chillag and S. Dolfi defined and studied so-called inverse semirational groups from a group theoretic perspective; it turned out that these groups are exactly the same groups as cut groups (cf. Proposition 2.1). The cut groups also play a role in other domains, and they have been taken up by several authors under different names, which exhibits a highly interesting interplay between group theory, representation theory, algebraic number theory and even $K$-theory (see e.g. [32, Section 3] for a complete survey).

One of the original reasons for studying cut groups is that $\mathcal{U}(\mathbb{Z} G)$ has a subgroup of finite index that is generated by the central units and the units of reduced norm one for all finite groups $G$. For many $G$, the latter group is generated by very specific unipotent units (called bicyclic units). These, together with the Bass units (a natural generalization of cyclotomic units), "determine" finitely many generators of the center. It is thus a natural question to determine when the central units can be avoided to determine finitely many generators of a large subgroup of $U(\mathbb{Z} G)$, i.e. characterize the cut groups. The problem of finding such a finite set of generators of large subgroups of $U(\mathbb{Z} G)$ is a vibrant topic since several decades; for details and many references, we refer to [28, Chapter 11].

Due to the fact that the rank of the center of $\mathcal{U}(\mathbb{Z} G)$ bounds the rank of its abelianization, and the latter is an important obstruction for certain fixed-point properties (like Serre's property (FA)), interest in cut groups arose recently from yet another perspective. This connection was used in [5] and [6] to describe when $\mathcal{U}(\mathbb{Z} G)$ has certain fixed-point properties and when it can be decomposed as a non-trivial amalgamated product up to commensurability. The relation between the abelianization and the center of $\mathcal{U}(\mathbb{Z} G)$ was further examined in [7].

Many interesting properties of cut groups are known. For instance, it follows from Higman's results [22] that an abelian group $G$ is cut if and only if its exponent divides 4 or 6 . The work of Ritter and Sehgal [38] yields that the cut property is quotient closed. It is observed by G. K. Bakshi, S. Maheshwary and I. B.S. Passi [8] that the center of a cut group is again a cut group, yet the cut property is not preserved under taking direct products. They also proved that every non-trivial cut group has order divisible by 2 or 3 . Furthermore, it is known that primes dividing the order of cut groups in certain classes are strongly restricted 
$[3,8,14,31]$, e.g. A. Bächle proved that only the primes $2,3,5$ and 7 divide the order of a solvable cut group [3]. Also, explicit descriptions of all cut groups in various classes of groups are obtained in these articles.

In the present work, we continue to examine the class of finite cut groups. After setting up the necessary background in Section 2, we investigate varied global and local properties of cut groups. In Section 3, we observe the impact of the cut property on character tables and show that the natural actions of a Galois group on the rows and the columns of the character table of a cut group are basically the same. Precisely, in Theorem 3.1, we prove the following.

Theorem A. Let $G$ be a cut group of exponent dividing $n$, and let $\zeta$ be a primitive $n$-th root of unity. Then the natural actions of $\operatorname{Gal}(\mathbb{Q}(\zeta) / \mathbb{Q})$ on the conjugacy classes and on the irreducible characters of $G$ are permutation isomorphic. In particular, the number of rational irreducible characters of $G$ is equal to the number of rational conjugacy classes of $G$.

Motivated by an element-wise criterion given in [31], for a nilpotent group of class 2 to be cut, we give a short proof of an easy element-free criterion in Section 4. This section is also supplemented with certain explicit and non-trivial examples of nilpotent cut groups.

Recently, it has been observed that an infinite simple group is always a cut group [9]. However, this is not true for finite simple groups. In Theorem 5.1, we give a complete list of finite simple cut groups.

Theorem B. Let $G$ be a (finite) simple group. Then $G$ is cut if and only if it is isomorphic to one of the following groups:

(1) $C_{2}, C_{3}$,

(2) $A_{7}, A_{8}, A_{9}, A_{12}$,

(3) $L_{2}(7), U_{3}(3), U_{3}(5), U_{4}(3), U_{5}(2), U_{6}(2), S_{4}(3), S_{6}(2), O_{8}^{+}(2)$,

(4) $M_{11}, M_{12}, M_{22}, M_{23}, M_{24}, C o_{1}, C o_{2}, C o_{3}, H S, M c L, T h, M$.

Further, in Section 6, we explore some local properties of cut groups: the impact of the cut property on $p$-subgroups. In particular, we prove that Sylow 3-subgroups of cut groups are again cut, for several classes of groups; see Proposition 6.4 and Theorem 6.6.

Theorem C. Let $G$ be a cut group and $P \in \operatorname{Syl}_{3}(G)$. Then $P$ is also cut, provided one of the following holds:

(1) $P$ is abelian,

(2) $P$ is a normal subgroup of $G$, 
(3) $G$ is supersolvable,

(4) $G$ is a Frobenius group,

(5) $G$ is simple,

(6) $G$ is of odd order and $\mathrm{O}_{3}(G)$ is abelian.

Finally, in Section 7, we present surprising data on the existence of cut groups. For instance, $86.62 \%$ of all groups up to order 512 are cut.

\section{Notation and preliminaries}

Throughout the article, all groups considered are finite, unless otherwise stated explicitly. Our notation is mostly standard; see $[24,28]$. Let $G$ be a group, and let $x \in G$ be an element of $G$. The order of $G$ is denoted by $|G|$, the order of $x$ is denoted by $o(x)$, and $\mathrm{C}_{G}(x)$ denotes the centralizer of $x$ in $G$. Let $y \in G$. Then, by $x \sim y$, we mean that " $x$ is conjugate to $y$ in $G$ ", i.e. $x^{g}:=g^{-1} x g=y$ for some $g \in G$, and $x^{G}$ denotes the conjugacy class of $x$ in $G$. The commutator subgroup of $G$ generated by all commutators $[x, y]=x^{-1} y^{-1} x y, x, y \in G$ is written as $G^{\prime}$. By $H \leqslant G(H \preccurlyeq G)$, we indicate that $H$ is a subgroup (normal subgroup) of $G$. For $H \leqslant G, \mathrm{~N}_{G}(H)$ and $[G: H]$, respectively, denote the normalizer and the index of $H$ in $G$. If $p$ is a prime, then $\operatorname{Syl}_{p}(G)$ is the set of Sylow $p$-subgroups of $G$. By $\operatorname{Irr}(G)$, we denote the set of irreducible complex characters of $G$, and for $\chi \in \operatorname{Irr}(G)$, by $\mathbb{Q}(\chi)$, we denote the field extension of the rationals generated by the values of the character $\chi$. Likewise, $\mathbb{Q}(x)$ denotes the field extension of the rationals generated by the values of all the characters of $G$ at the element $x \in G$.

An element $x \in G$ is called rational in $G$ if $x^{j} \sim x$ for all $j$ coprime to $o(x)$ or, equivalently, $\mathbb{Q}(x)=\mathbb{Q}$; see e.g. [24, Problem (2.12)]. Of course, a conjugacy class $x^{G}$ of $G$ is called rational if it consists of elements rational in $G$. Likewise, we say a character $\chi$ of $G$ is rational if $\mathbb{Q}(\chi)=\mathbb{Q}$. The group $G$ is called rational if every element $x \in G$ is rational in $G$ or, equivalently, every $\chi \in \operatorname{Irr}(G)$ is rational.

We briefly mention certain classes of groups that are closely related to cut groups. A character $\chi$ of $G$ is called quadratic if $[\mathbb{Q}(\chi): \mathbb{Q}]=2$. If every irreducible character of the group $G$ is either rational or quadratic, then it is said to be quadratic rational. Following [14], an element $x \in G$ is called semi-rational in $G$ if there exists an integer $m$ such that $x^{j} \sim x$ or $x^{j} \sim x^{m}$ for every $j \in \mathbb{Z}$ coprime to $o(x)$, and $x$ is called inverse semi-rational in $G$ if $m=-1$, i.e. $x^{j} \sim x$ or $x^{j} \sim x^{-1}$ for every such $j$. The group $G$ is called (inverse) semi-rational if every element of $G$ is (inverse) semi-rational in $G$. From the definition, it is clear that rational groups are inverse semi-rational. 
We begin by stating some of the well-known equivalent criteria for a cut group that are essential for this article.

Proposition 2.1 (see [3, Proposition 2.2], [32, Theorem 5]). For a group G, the following statements are equivalent.

(1) $G$ is a cut group, i.e. $\mathbb{Z}(\mathcal{U}(\mathbb{Z} G))= \pm \mathbb{Z}(G)$.

(2) $G$ is an inverse semi-rational group, i.e. for every $x \in G$ and $j \in \mathbb{Z}$ with $j$ coprime to $o(x), x^{j} \sim x$ or $x^{j} \sim x^{-1}$.

(3) If $\mathbb{Q} G \simeq \bigoplus_{k=1}^{m} M_{n_{k}}\left(D_{k}\right)$ is the Wedderburn decomposition of the rational group algebra $\mathbb{Q} G\left(m, n_{k} \in \mathbb{Z} \geqslant 1, D_{k}\right.$ division algebras $)$, then for each $k$, $\mathcal{Z}\left(D_{k}\right) \simeq \mathbb{Q}(\sqrt{-d})$ for some $d=d(k) \in \mathbb{Z}_{\geqslant 0}$.

(4) For each $\chi \in \operatorname{Irr}(G), \mathbb{Q}(\chi)=\mathbb{Q}(\sqrt{-d})$ for some $d=d(\chi) \in \mathbb{Z} \geqslant 0$.

In particular, the notion of a cut group and an inverse semi-rational group are the same. To avoid confusion, from now on, we use the term inverse semi-rational for elements of the group and the term cut for a group. Note that the above proposition implies that every element of order 1,2,3,4 or 6 is inverse semi-rational in every group it is contained in; in particular, all groups of exponent a divisor of 4 or 6 are cut.

There are other known equivalent characterizations of cut groups (for example, a group $G$ is cut if and only if the Whitehead group $K_{1}(\mathbb{Z} G)$ is finite); cf. [32, Theorem 5]. We will give yet another one in Proposition 3.2 below. The cut groups are in the intersection of quadratic rational and semi-rational groups. Observe that the dihedral group of order 16 is both, quadratic rational and semi-rational, yet it is not cut. However, we shall see in Section 7 that cut groups constitute a big part of this intersection.

A well-known and very useful fact is that the cut property is inherited by quotients. There are short proofs (e.g. in [14, Lemma 4]) of this fact using any of the equivalent statements of cut groups in Proposition 2.1 with the exception of the very definition. We take this opportunity to provide a short $\mathbb{Z} G$-proof that avoids passing through any of the characterizations in Proposition 2.1.

Lemma 2.2. Let $G$ be a cut group and $N \leqslant G$. Then $G / N$ is a cut group.

Proof. Let $R$ be a subring of a ring $T$ with the same identity. Suppose $I \subseteq R$ and $I$ is an ideal of $T$. Assume $\mathcal{U}(T / I)$ is a torsion group. If $\mathcal{Z}(\mathcal{U}(R))$ is torsion, then $\mathcal{Z}(\mathcal{U}(T))$ is torsion: take $v \in \mathcal{Z}(\mathcal{U}(T))$; then $\bar{v}^{n}=1$ and $\bar{v}^{-n}=1$ for some positive integer $n$, where bars indicate reduction modulo $I$. Hence,

$$
v^{n}-1, v^{-n}-1 \in I \subseteq R .
$$


So $v^{n}, v^{-n} \in R$, and thus $v^{n} \in \mathcal{Z}(\mathcal{U}(R))$. Since $\mathcal{Z}(\mathcal{U}(R))$ is torsion, it follows that $v \in \mathcal{Z}(U(T))$ has finite order and $\mathcal{Z}(U(T))$ is a torsion group.

Apply this to

$$
R=\mathbb{Z} G \subseteq T=\mathbb{Z} G \cdot \hat{N} \oplus \mathbb{Z} G \cdot(1-\hat{N})(\subseteq \mathbb{Q} G),
$$

where $\hat{N}=\frac{1}{|N|} \sum_{n \in N} n$, a central idempotent of $\mathbb{Q} G$. Clearly, $I=|N| \cdot T$ is a common ideal of $T$ and $R$, and $T / I$ is finite. Thus, $U(T / I)$ is a finite group. Note that $\mathbb{Z} G \cdot \hat{N}$ and $\mathbb{Z}[G / N]$ are isomorphic as unital rings. Hence, if $G$ is a cut group, then $\mathcal{Z}(\mathcal{U}(R))$ is finite, and by the above, $\mathcal{Z}(\mathcal{U}(T))$ is torsion, and hence all central units of $\mathbb{Z} G \cdot \hat{N} \simeq \mathbb{Z}[G / N]$ are torsion. By a corollary to the BermanHigman theorem [28, Corollary 7.1.4(2)], all torsion central units of an integral group ring of a finite group are trivial. Thus, $G / N$ is a cut group.

\section{Rationality}

It is a major problem in the representation theory of finite groups to detect dualities between conjugacy classes and irreducible characters of groups. In general, the number of rational conjugacy classes and rational irreducible characters of a group $G$ do not agree ${ }^{1}$ : there are groups as small as order 32 such that the number of rational conjugacy classes exceeds the number of rational irreducible characters and vice versa; see Example 3.3 below. However, for interesting classes, these numbers do coincide, and it is a popular theme to detect group or representation theoretic conditions that guarantee this. For instance, G. Navarro and P. H. Tiep proved that this is true for groups with at most two rational irreducible characters [36, Corollary 9.7, Theorem A] (these proofs require the Classification of Finite Simple Groups). Also, for groups with cyclic Sylow 2-subgroups, the number of rational conjugacy classes and rational characters coincide, as shown by Navarro and J. Tent [35]. Note that a group with a cyclic Sylow 2-subgroup $P$ can only be cut if $|P| \leqslant 4$, by Cayley's normal 2-complement theorem and Lemma 2.2.

For a structural explanation of such phenomena, one might want to consider a suitable group that acts naturally on both sets in question. Assume that $G$ is a group of exponent dividing $n$, and let $\zeta$ be a fixed primitive complex $n$-th root of unity. Then the elements of the Galois group $\operatorname{Gal}(\mathbb{Q}(\zeta) / \mathbb{Q})$ are determined by $\sigma: \zeta \mapsto \zeta^{m}$ for an integer $m=m(\sigma)$ coprime to $n$, unique modulo $n$. This induces an isomorphism $\operatorname{Gal}(\mathbb{Q}(\zeta) / \mathbb{Q}) \rightarrow U(\mathbb{Z} / n \mathbb{Z}): \sigma \mapsto m$. Now $\operatorname{Gal}(\mathbb{Q}(\zeta) / \mathbb{Q})$

\footnotetext{
${ }^{1}$ Rational conjugacy classes should not be confused with so-called $\mathbb{Q}$-conjugacy classes (or $\mathbb{Q}$-classes); see [28, p. 231] for a definition: the former are actual conjugacy classes of $G$, whereas the latter are in general unions of conjugacy classes of $G$, similarly for rational irreducible characters and irreducible $\mathbb{Q}$-characters. In fact, the number of $\mathbb{Q}$-classes and irreducible $\mathbb{Q}$-characters always coincide by a theorem of Artin [28, Corollary 7.1.12].
} 
acts naturally on the irreducible characters $\operatorname{Irr}(G)$ of $G$ and the conjugacy classes $\operatorname{ccl}(G)$ of $G$ by

$$
\chi^{\sigma}=\sigma^{-1} \circ \chi, \quad\left(x^{G}\right)^{\sigma}=\left(x^{m}\right)^{G}, \quad \chi \in \operatorname{Irr}(G), x \in G, \sigma \in \operatorname{Gal}(\mathbb{Q}(\zeta) / \mathbb{Q}) .
$$

Note that the fixed points of these actions are precisely the rational irreducible characters and the rational conjugacy classes, respectively. One might wonder under which conditions these actions are essentially the same, i.e. in which situations they are permutation isomorphic: recall that two actions of a group $\Gamma$ on sets $X$ and $Y$ are called permutation isomorphic if there is a bijection $\alpha: X \rightarrow Y$ such that $\alpha\left(x^{g}\right)=\alpha(x)^{g}$ for all $x \in X$ and all $g \in \Gamma$. The above actions are permutation isomorphic for $p$-groups of odd order (follows from Brauer's permutation lemma [24, Theorem (6.32)]) and also for groups all whose Sylow subgroups are abelian [13]. Here we show that this also happens for cut groups. In view of the group theoretic characterization of cut groups in Proposition 2.1 (2), this can be seen as a contribution to [34, (14.4) Problem].

Theorem 3.1. Let $G$ be a cut group of exponent dividing $n$, and let $\zeta$ be a primitive $n$-th root of unity. Then the natural actions of $\operatorname{Gal}(\mathbb{Q}(\zeta) / \mathbb{Q})$ on the conjugacy classes and on the irreducible characters of $G$ are permutation isomorphic. In particular, the number of rational irreducible characters of $G$ is equal to the number of rational conjugacy classes of $G$.

Proof. We start with the following group theoretic fact.

Claim. Assume a (finite) group $\Gamma$ acts on two finite sets $X$ and $Y$ of the same size and every orbit of $\Gamma$ on both sets has length at most 2 . If every element of $\Gamma$ has the same number of fixed points on $X$ as it has on $Y$, then the actions are permutation isomorphic.

Denote by $f(\sigma)$ the number of fixed points of $\sigma \in \Gamma$ on $X$ or, equivalently, on $Y$. By a classical result on permutation actions (see [24, Lemma (13.23)]), it suffices to show that each $\Delta \leqslant \Gamma$ has the same number of fixed points on $X$ as it has on $Y$ to conclude that the actions are permutation isomorphic. Arguing by induction on $|\Gamma|$, it is enough to show that $\Gamma$ has the same number of fixed points on $X$ as it has on $Y$. By Burnside's lemma [40, Theorem 3.22], the number $k$ of orbits of $\Gamma$ on $X$ or on $Y$ is

$$
k=\frac{1}{|\Gamma|} \sum_{\sigma \in \Gamma} f(\sigma) .
$$

Thus, the number of fixed points on both sets is just $2 k-|X|=2 k-|Y|$. This proves the claim. 
Set $\Gamma=\operatorname{Gal}(\mathbb{Q}(\zeta) / \mathbb{Q})$. By Proposition $2.1(4)$, we have for each $\chi \in \operatorname{Irr}(G)$ that $\mathbb{Q}(\chi)=\mathbb{Q}(\sqrt{-d})$ for some $d \in \mathbb{Z} \geqslant 0$. So the length of each orbit of the natural action of $\Gamma$ on $X=\operatorname{Irr}(G)$ is at most 2. By Proposition 2.1 (2), the length of each orbit of the natural action of $\Gamma$ on $Y=\operatorname{ccl}(G)$ is at most 2. We want to employ Brauer's permutation lemma [24, Theorem (6.32)] to obtain that each $\sigma \in \Gamma$ has the same number of fixed points on $X$ as it has on $Y$. To do this, we need to verify that $\chi^{\sigma}\left(x^{\sigma}\right)=\chi(x)$ for all $\chi \in \operatorname{Irr}(G)$ and all $x \in G$. This can be checked by the following calculation. Let $D$ be a representation with character $\chi$. Then $D(x)$ is conjugate to $\operatorname{diag}\left(\rho_{1}, \ldots, \rho_{d}\right)$, a diagonal matrix with $n$-th roots of unity $\rho_{1}, \ldots, \rho_{d}$ on its diagonal. Let $\sigma \in \Gamma$ and $m=m(\sigma)$ be as defined above. Then

$$
\begin{aligned}
\chi^{\sigma}\left(x^{\sigma}\right) & =\sigma^{-1} \circ \operatorname{Tr} D\left(x^{m}\right)=\sigma^{-1} \circ \operatorname{Tr} D(x)^{m} \\
& =\sigma^{-1} \circ \operatorname{Tr} \operatorname{diag}\left(\rho_{1}^{m}, \ldots, \rho_{d}^{m}\right)=\sigma^{-1}\left(\rho_{1}^{m}+\cdots+\rho_{d}^{m}\right) \\
& =\rho_{1}+\cdots+\rho_{d}=\operatorname{Tr} \operatorname{diag}\left(\rho_{1}, \ldots, \rho_{d}\right)=\operatorname{Tr} D(x)=\chi(x) .
\end{aligned}
$$

Consequently, the natural actions of $\Gamma$ on $X$ and on $Y$ are permutation isomorphic by the above claim.

Since the actions of $\Gamma$ on the irreducible characters and on the conjugacy classes are permutation isomorphic, they have the same number of fixed points, so the claim about the rational-valued characters and conjugacy classes follows.

Let $G$ be a group of exponent $n$ and $\Gamma=\operatorname{Gal}(\mathbb{Q}(\zeta) / \mathbb{Q})$ for a primitive complex $n$-th root of unity $\zeta$. Denote by $N \leqslant \Gamma$ the kernel of the natural action of $\Gamma$ on $\operatorname{Irr}(G)$ (or on $\operatorname{ccl}(G)$ ). If $G$ is semi-rational or quadratic rational, then $\Gamma / N$ is an elementary abelian 2-group (see e.g. [14, Lemma 7]). If $G$ is a solvable semi-rational group (solvable quadratic rational group, respectively), then $|\Gamma / N|$ is bounded by $2^{6}$ (by $2^{7}$, respectively) by [42, Theorem B and Corollary 8]. In particular, for a solvable cut group $G$, one obtains that $\Gamma / N$ has order dividing $2^{5}$ by adapting the proof of [42, Theorem B] and using that $|G|$ is divisible by at most 4 different primes by [3, Theorem 1.2]. Many solvable cut groups such that $|\Gamma / N|=8$ can be found in the library of small groups in GAP [43]. For instance, the groups with SmallGroupIDs $[144,58],[192,718]$ and $[960,11363]$; the sets of fields of character values of their irreducible characters are, respectively,

$$
\begin{aligned}
& \{\mathbb{Q}, \mathbb{Q}(\sqrt{-1}), \mathbb{Q}(\sqrt{-2}), \mathbb{Q}(\sqrt{-3})\}, \\
& \{\mathbb{Q}, \mathbb{Q}(\sqrt{-1}), \mathbb{Q}(\sqrt{-3}), \mathbb{Q}(\sqrt{-6})\}, \\
& \{\mathbb{Q}, \mathbb{Q}(\sqrt{-1}), \mathbb{Q}(\sqrt{-3}), \mathbb{Q}(\sqrt{-15})\} .
\end{aligned}
$$

We do not know of an example of a solvable cut group such that $|\Gamma / N|$ exceeds $2^{3}$. Note that alternating groups are both, semi-rational and quadratic rational. So if 
we drop the solvability assumption, then [39] shows that $\Gamma / N$ can get arbitrarily large for semi-rational and quadratic rational groups. However, we do not know whether or not the order $|\Gamma / N|$ is bounded in the case of arbitrary cut groups (cf. [4, Question 1]).

We continue with yet another characterization of cut groups based on fields of character values that is dual to the one in Proposition 2.1 (4): a group $G$ is cut if and only if, for all irreducible characters $\chi$ of $G, \mathbb{Q}(\chi)$ is $\mathbb{Q}$ or imaginary quadratic.

Proposition 3.2. Let $G$ be a group. The following statements are equivalent.

(1) $G$ is a cut group.

(2) For each $x \in G, \mathbb{Q}(x)=\mathbb{Q}(\sqrt{-d})$ for some $d=d(x) \in \mathbb{Z}_{\geqslant 0}$.

Proof. Assume first that $G$ is a cut group. Let $x \in G$ so that $x$ is inverse semirational in $G$ and in particular semi-rational in $G$. From [42, Lemma 1], it thus follows that $[\mathbb{Q}(x): \mathbb{Q}] \leqslant 2$. Now, if $x$ is not rational, then for some $\chi \in \operatorname{Irr}(G)$, $\mathbb{Q} \neq \mathbb{Q}(\chi(x)) \subseteq \mathbb{Q}(x)$, and degree considerations yield $\mathbb{Q}(\chi(x))=\mathbb{Q}(x)$. Furthermore, $\mathbb{Q} \neq \mathbb{Q}(\chi(x)) \subseteq \mathbb{Q}(\chi)$, and by Proposition $2.1, \mathbb{Q}(\chi)=\mathbb{Q}(\sqrt{-d})$ for some $d>0$. Consequently, (2) follows.

Conversely, assume that, for each $x \in G$, we have $\mathbb{Q}(x)=\mathbb{Q}(\sqrt{-d}), d \geqslant 0$. Fix $x \in G$. Again by [42, Lemma 1], $x$ is semi-rational in $G$ so that there is $m \in \mathbb{Z}$ such that,

for every integer $j$ coprime to $o(x), \quad x^{j} \sim x$ or $x^{j} \sim x^{m}$.

Assume first $x \sim x^{-1}$. Then, for every $\chi \in \operatorname{Irr}(G)$, we have $\chi(x)=\chi\left(x^{-1}\right)=\overline{\chi(x)}$, where bar indicates complex conjugation. It follows that

$$
\chi(x) \in \mathbb{Q}(\sqrt{-d}) \cap \mathbb{R}=\mathbb{Q} \text { for all } \chi \in \operatorname{Irr}(G) .
$$

Thus, $\mathbb{Q}(x)=\mathbb{Q}$, i.e. $x$ is rational in $G$. Otherwise, $x$ is not conjugate to $x^{-1}$ in $G$, and in view of $(\mathrm{C}), x^{-1}$ is conjugate to $x^{m}$, and we actually may chose $m=-1$ in (C). Hence, in any case, $x$ is inverse semi-rational in $G$ and $G$ is a cut group (Proposition 2.1).

Note that Theorem A cannot be extended to the slightly larger class of semirational groups nor to the class of quadratic rational groups. A reason for this is that the nice symmetry between the rows and the columns of the character table in the characterization of cut groups (Propositions 2.1 (4) and 3.2) does not hold true for these classes of groups. Indeed, by [42, Lemma 1], a group is semi-rational if and only if $[\mathbb{Q}(x): \mathbb{Q}] \leqslant 2$ for all $x \in G$. However, the degree of the dual field extensions $\mathbb{Q}(\chi)$ over $\mathbb{Q}$ can get large, e.g. for the semi-rational group $G$ with SmallGroupID [384, 3283] in GAP [43], there is $\chi \in \operatorname{Irr}(G)$ such that $\mathbb{Q}(\chi)=\mathbb{Q}(\zeta)$ for 
a primitive 24-th root of unity $\zeta$. Similarly, one can find examples of a quadratic rational groups $G$ such that the fields $\mathbb{Q}(x), x \in G$, get large. The following example shows that, already for groups of order 32, the actions of the Galois group on the rows and columns of the character table need not be permutation isomorphic and even that the numbers of their fixed points might differ.

Example 3.3. In [42, Section 6], Tent defined a semi-rational group $G$ and a quadratic rational group $H$ (the latter originally constructed by E. Dade; see [13, Example 3.6]) as follows:

$$
\begin{aligned}
& G=\left\langle a, b, c \mid a^{2}=b^{2}=c^{8}=1, b^{c}=b, b^{a}=b c^{4}, c^{a}=c^{3}\right\rangle, \\
& H=\left\langle a, b, c \mid a^{2}=b^{2}=c^{8}=1, b^{c}=b, b^{a}=b, c^{a}=b c^{3}\right\rangle
\end{aligned}
$$

(having GAP SmallGroupIDs [32, 42] and [32, 9], respectively). If we denote by $\operatorname{Irr}_{\mathbb{Q}}(G)$ and $\operatorname{ccl}_{\mathbb{Q}}(G)$ the set of rational irreducible characters and rational conjugacy classes of $G$, respectively, then we have $\left|\operatorname{Irr}_{\mathbb{Q}}(G)\right|=10>\left|\operatorname{ccl}_{\mathbb{Q}}(G)\right|=8$ and $\left|\operatorname{Irr}_{\mathbb{Q}}(H)\right|=6<\left|\operatorname{ccl}_{\mathbb{Q}}(H)\right|=8$. These are examples of smallest possible order for which these quantities differ.

\section{Nilpotent cut groups}

As mentioned in the introduction, it is well known that an abelian group is cut if and only if its exponent divides 4 or 6 . As the cut property is quotient closed (Lemma 2.2) and the center of a cut group is again a cut group, we necessarily have, for each cut group $G$,

$$
\text { for all } N \leqslant G, \quad \exp (\mathcal{Z}(G / N)) \mid 4 \quad \text { or } \quad \exp (\mathcal{Z}(G / N)) \mid 6,
$$

where $\exp (H)$ denotes the exponent of a group $H$. However, rarely, condition $(\mathrm{N})$ is sufficient for $G$ to be a cut group. For example, (N) holds for all non-abelian simple groups, but not all simple groups are cut (see Section 5). Also, dihedral groups of order $2 p, p$ a prime, satisfy $(\mathrm{N})$, but these groups are not cut for $p \geqslant 5$ (and are of derived length 2). In [8], it was noted that a nilpotent cut group is always a $\{2,3\}$-group, and an element-wise criterion for a nilpotent group to be a cut group was provided. In the case of nilpotency class 2, the criterion was refined in [31]. For a nilpotent group $G$ of class 2 to be a cut group, we provide a characterization on the quotients of $G$. We prove that, for such groups, condition $(\mathrm{N})$ is sufficient for $G$ to be a cut group.

After we had finished writing the first version of this article we were informed by V. Bovdi that such a characterization was already obtained by Z. Pată and 
A. Bovdi [12,37]. The formulation of [12, Theorem 8.2] gave the inspiration for the new group theoretic proof below that is notably shorter than the ring theoretic proof presented in [12]. As it seems to be hard to get hold of the above sources, being available in Ukrainian and Russian only, and since the result seems to have stayed unnoticed by many experts, we want to take the opportunity to popularize this theorem together with our concise proof below.

Theorem 4.1 (Pată). Let $G$ be a nilpotent group of class at most 2 . Then $G$ is cut if and only if the exponent of $\mathcal{Z}(G / N)$ divides 4 or 6 for all $N \leqslant G$. Actually, it is sufficient to deal with normal subgroups $N$ of the form $[g, G]$.

Proof. Clearly, the condition is necessary. For sufficiency, assume that $G$ is a finite nilpotent group of class at most 2 and the exponent of $Z(G / N)$ divides 4 or 6 for all $N \leqslant G$. The nilpotency of $G$ yields that $G$ is a $\{2,3\}$-group and $G=P_{2} \times P_{3}$, where $P_{2}$ and $P_{3}$ denote its Sylow 2- and 3-subgroups, respectively. In view of [31, Theorem 3], $G$ is a cut group if and only if both $P_{2}$ and $P_{3}$ are cut groups, and moreover, $P_{2}$ is rational if $P_{3}$ is non-trivial. Since $G$ is of class at most $2,[h, G]=\{[h, g]: g \in G\}$ is a normal subgroup of $G$ for any $h \in G$. We first check that $P_{2}$ is a cut group. By [31, Corollary 3], this is equivalent with $x^{4} \in\left[x, P_{2}\right]=[x, G]$ for all $x \in P_{2}$. But this follows from the assumptions since the image of $x$ is contained in $\mathcal{Z}(G /[x, G])$. Similarly, $P_{3}$ is also a cut group. Hence, it only remains to show that if $P_{3}$ is non-trivial, then $P_{2}$ is rational. Assume that $P_{3} \neq\{1\}$, and take $x \in P_{2}$. Clearly, $[x, G]=\left[x, P_{2}\right] \leqslant P_{2}$, and 3 divides the order of $\mathcal{Z}(G /[x, G])$. Then, by the assumption, $x^{6} \in[x, G]$, and thus $x^{2} \in[x, G]$. Hence, for any odd integer $j=2 v+1$, we have

$$
x^{j}=x\left(x^{2}\right)^{v} \in x[x, G]=x\left[x, P_{2}\right],
$$

and thus $x^{j} \in x^{P_{2}}$. So $x$ is rational in $P_{2}$, as desired.

Remark 4.2. The following two groups of nilpotency class 3 illustrate that condition (N) is no longer sufficient to conclude that $G$ is cut for nilpotent groups of nilpotency class exceeding 2 . First, let $G=D_{16}$, the dihedral group of order 16 . Then we have $\exp (\mathcal{Z}(G / N)) \mid 2$ for every normal subgroup, yet the group is not cut. Similarly, if

$$
\begin{aligned}
G & =\left\langle a, b, c \mid a^{9}=b^{3}=c^{3}=1, b^{a}=b, a^{c}=a b, b^{c}=a^{3} b\right\rangle \\
& \simeq\left(C_{9} \times C_{3}\right) \rtimes C_{3},
\end{aligned}
$$

(SmallGroupID $[81,8]$ ), all centers of quotients of $G$ are of exponent 3 , but this group is not cut. 
There is a plethora of nilpotent cut groups of small exponent; see Proposition 7.1 and its proof. Here we provide further examples of nilpotent cut groups, in particular some of arbitrary large exponent and derived length (and hence nilpotency class).

Example 4.3. (1) Sylow $p$-subgroups of $S_{n}$ for all $n, p \in\{2,3\}$. The Sylow 2and 3-subgroups of symmetric groups $S_{n}$ are rational and cut, respectively, as follows from [14, Remark 14]. We provide some explanation here. The structure of the Sylow $p$-subgroups of the symmetric group $S_{n}$ is well known; see [40, Theorem 7.27] and the discussion following it. Denote by $Q_{n}$ a Sylow 2-subgroup of $S_{n}$. First assume that $n$ is a power of 2 . Then $Q_{n}=\left(\left(C_{2} \succ C_{2}\right) \ldots \succ C_{2}\right)<C_{2}$ is an iterated wreath product of cyclic groups of order 2. Clearly, $C_{2}$ is rational and so is $Q_{n}$ by [21, Proposition 3.5]. Now if $n$ is arbitrary, consider its base-2 expansion $n=a_{k} \cdot 2^{k}+a_{k-1} \cdot 2^{k-1}+\cdots+a_{1} \cdot 2+a_{0}$ with each $a_{\ell} \in\{0,1\}$. Then the Sylow 2-subgroup of $S_{n}$ is

$$
Q_{n}=Q_{2^{k}}^{a_{k}} \times Q_{2^{k-1}}^{a_{k-1}} \times \cdots \times Q_{2}^{a_{1}} .
$$

Since the direct product of rational groups is rational again, $Q_{n}$ is a rational group for all $n$.

Similarly, the Sylow 3-subgroups $R_{n}$ of $S_{n}$ are iterated wreath products of $C_{3}$ if $n$ is a power of 3. Since $\mathbb{Q}(\chi) \subseteq \mathbb{Q}(\sqrt{-3})$ for all $\chi \in \operatorname{Irr}\left(C_{3}\right)$, we also have that, for these iterated wreath products, $\mathbb{Q}(\psi) \subseteq \mathbb{Q}(\sqrt{-3})$ for all $\psi \in \operatorname{Irr}\left(R_{n}\right)$ by repeated application of [21, Proposition 3.5] (see also the remark before the definition preceding that proposition), so they are cut groups. In the general setting, the Sylow 3-subgroups $R_{n}$ of $S_{n}$ are also cut as direct products of these iterated wreath products by [8, Corollary 1]. Note that the exponent and derived length of $Q_{n}$ and $R_{n}$ grow beyond all limits.

(2) Sylow p-subgroups of $A_{n}$ for all $n, p \in\{2,3\}$. Also, the Sylow 2- and 3subgroups of alternating groups $A_{n}$ are rational and cut, respectively. Since $A_{n}$ has index 2 in $S_{n}$, the Sylow 3 -subgroups of $A_{n}$ are cut by (1) above. We now show that the Sylow 2-subgroups of alternating groups are rational. For this, let $Q_{n}$ be again a Sylow 2-subgroup of $S_{n}$. Then $P_{n}=Q_{n} \cap A_{n}$ is a Sylow 2-subgroup of $A_{n}$. Let $x \in P_{n}$, and let $j$ be an odd integer. We need to show that there is $y \in P_{n}$ such that $x^{y}=x^{j}$. Write $x=x_{k} \cdot \ldots \cdot x_{1}$ with $x_{\ell} \in Q_{2} \ell$ according to the decomposition in (D). Now $x_{\ell} \in Q_{2^{\ell}}$ is conjugate by a suitable $y_{\ell} \in Q_{2^{\ell}}$ to $x_{\ell}^{j}$ since $Q_{2^{\ell}}$ is rational. Note that we may choose $y_{\ell}$ as an odd permutation (in the case $y_{\ell}$ is even and $x_{\ell} \neq 1$, decompose $x_{\ell}=t_{1} \cdot \ldots \cdot t_{s}$ in non-identity disjoint cycles of $Q_{2} \ell$ of 2-power length; then $y_{\ell}^{\prime}=t_{1} y_{\ell} \in Q_{2^{\ell}}$ is an odd permutation such that $x_{\ell}^{y_{\ell}^{\prime}}=x_{\ell}^{j}$ ). Then, for $y \in P_{n}$, the product of all these $y_{\ell}$, we have $x^{y}=x^{j}$. 
If $n=4 m+2$ or $n=4 m+3$ for some $m \in \mathbb{Z}_{\geqslant 1}$, then $S_{4 m}$ embeds into $A_{4 m+2}$ and $A_{4 m+3}$, and the image has odd index

$$
(2 m+1)(4 m+1) \text { and }(2 m+1)(4 m+1)(4 m+3),
$$

respectively, so their Sylow 2-subgroups are isomorphic. However, in all the other cases, order considerations show that the Sylow 2-subgroups of $A_{n}$ can never be isomorphic to a Sylow subgroup of a symmetric group, so they are genuinely different examples of rational nilpotent groups of large derived length and exponent.

(3) Some Sylow p-subgroups of linear groups. To indicate that it might be difficult to decide whether a group is cut or not, even for intensively studied groups, we consider for instance the group of unipotent upper triangular matrices over finite fields. Let $p$ be a prime, and let $n$ and $f$ be positive integers. Denote by $P$ the Sylow $p$-subgroup of $\operatorname{GL}\left(n, p^{f}\right)$ consisting of unipotent upper triangular matrices. Note that $P$ is also isomorphic to a Sylow $p$-subgroup of $\operatorname{PSL}\left(n, p^{f}\right)$. It was believed for a long time and supported by experimental calculations that, for $p=2$, the group $P$ is a rational group for all $n$. Only in 1998, I. M. Isaacs and D. Karagueuzian, in $[25,26]$, gave an example of a matrix $A \in P$ that is not conjugate to its inverse for $n=13$ and $p^{f}=2$. Actually, the Sylow $p$-subgroups of $\operatorname{GL}\left(n, p^{f}\right)$ are rational if and only if $p=2$ and $n \leqslant 12$ (see [33, Introduction]). Also, the Sylow 3-subgroups of $\operatorname{GL}\left(n, 3^{f}\right)$ are cut for all $n \leqslant 12$; see [33, Introduction]. However, it seems to be still unknown when exactly the Sylow $p$-subgroups of $\operatorname{GL}\left(n, p^{f}\right)$ are cut. If they are, then $(p=2$ and $n \leqslant 48)$ or $(p=3$ and $n \leqslant 18$ ) by [33, Theorem].

For some other simple groups, it is verified in the proof of Theorem 6.6(3) that their Sylow 3-subgroups are cut.

Question 4.4. Which Sylow 2- and 3-subgroups of classical groups or groups of Lie type are cut?

Remark 4.5. It is well known that every $\{2,3\}$-group can be embedded into a rational $\{2,3\}$-group; see e.g. [19, Proposition 1]. Not every nilpotent $\{2,3\}$-group can be embedded into a nilpotent rational group though for trivial reasons (every non-trivial rational group has even order). However, Example 4.3 (1) shows that every nilpotent $\{2,3\}$-group can be embedded into a nilpotent cut group.

The set $\pi=\{2,3\}$ above is optimal for the embedding property for solvable $\pi$-groups into rational solvable groups: every rational solvable group is a $\{2,3,5\}$ group by a classical result of R. Gow [19] and has an elementary abelian Sylow 5-subgroup by work of P. Hegedús [21], so the structure of the Sylow 5-subgroup is a proper obstruction. 
However, for solvable cut groups, we show next that the obstruction in Remark 4.5 is no longer an obstruction for the corresponding embedding question. Recall [3, Theorem 1.2] that the order of a solvable cut group can only be divisible by the primes $2,3,5$ and 7 .

Proposition 4.6. Every 5- and every 7-group can be embedded into a solvable cut group.

Proof. We work the proof out for $p=5$. Consider $P=\langle(1,2,3,4,5)\rangle \leqslant S_{5}$, a Sylow 5-subgroup of $S_{5}$, and set

$$
W_{5}=\mathrm{N}_{S_{5}}(P)=\langle(1,2,3,4,5),(2,3,5,4)\rangle \leqslant S_{5},
$$

its normalizer in $S_{5}$. Then $W_{5} \simeq C_{5} \rtimes C_{4}$, the Frobenius group of order 20. Note that $\mathbb{Q}(\chi) \subseteq \mathbb{Q}(i)$ for all $\chi \in \operatorname{Irr}\left(W_{5}\right)$, and hence $W_{5}$ is a solvable cut group. Define $W_{5^{n+1}}=W_{5^{n}} 2 W_{5}, n \in \mathbb{Z}_{\geqslant 1}$. Induction and [21, Proposition 3.5] show that $W_{5^{n}}$ is a cut group for all $n \in \mathbb{Z} \geqslant 1$ which is clearly solvable as wreath product of solvable groups. Note that the Sylow 5 -subgroup of $W_{5^{n}}$ is isomorphic to a Sylow 5 -subgroup of $S_{5^{n}}$. Since every 5-group can be embedded into a symmetric group of sufficiently large degree, by Cayley's theorem, it can also be embedded into some $S_{5^{n}}$ and hence into the solvable cut group $W_{5^{n}}$.

For $p=7$, one can argue similarly using $W_{7}=\mathrm{N}_{S_{7}}(\langle(1,2,3,4,5,6,7)\rangle) \leqslant S_{7}$, which is isomorphic to the Frobenius group of order 42.

Question 4.7. Can every solvable $\{2,3,5,7\}$-group be embedded into a solvable cut group?

\section{Simple cut groups}

So far, the properties of solvable cut groups have been explored. A complete classification of finite metacyclic cut groups is given in [8, Theorem 5]. A description of Frobenius cut groups can be found in [3, Theorem 1.3] (it turns out that those groups are always solvable). In this section, we give a complete classification of finite simple cut groups based on work of S. H. Alavi and A. Daneshkhah.

Theorem 5.1. Let $G$ be a simple group. Then $G$ is cut if and only if it is isomorphic to one of the following groups:

(1) $C_{2}, C_{3}$,

(2) $A_{7}, A_{8}, A_{9}, A_{12}$,

(3) $L_{2}(7), U_{3}(3), U_{3}(5), U_{4}(3), U_{5}(2), U_{6}(2), S_{4}(3), S_{6}(2), O_{8}^{+}(2)$,

(4) $M_{11}, M_{12}, M_{22}, M_{23}, M_{24}, C o_{1}, C o_{2}, C o_{3}, H S, M c L, T h, M$. 
Proof. The simple semi-rational groups are classified in [1, Theorem 1.1] using the Classification of Finite Simple Groups. (Note that the groups in the statement of [1, Theorem 1.1] are not entirely correctly listed: the groups ${ }^{3} D_{4}(2),{ }^{3} D_{4}(3)$, ${ }^{2} B_{2}(8),{ }^{2} B_{2}(32),{ }^{2} G_{2}(27)$ and the Tits group ${ }^{2} F_{4}(2)^{\prime}$ are there by accident, and the group $G_{2}(4)$ is missing, yet the proofs in the article [1] correctly identify the former groups as not being semi-rational and the latter group as semi-rational.) Disregarding the alternating groups, this is a finite list. The alternating groups that are cut are described in $[2,18]$. An inspection of the character tables of the remaining groups (for example, in ATLAS [15] or GAP) using Proposition 2.1 reveals that of those exactly the groups listed above are cut groups.

Note that, in contrast to the case of finite simple groups, every infinite simple group $I$ is a cut group, i.e. has the property that $\mathbb{Z}(\mathcal{U}(\mathbb{Z} I))= \pm \mathbb{Z}(I)$; see $[9$, Examples following Theorem 5].

\section{Local properties of cut groups}

It was conjectured for a long time that being rational for 2-elements is governed by the Sylow 2-subgroup of a group. More precisely, already in Kletzing's book from 1984 [30, p. 13], it is referred to as a "long standing conjecture" that the Sylow 2-subgroup of a rational group is again rational (recall that every non-trivial rational group is of even order and hence has a non-trivial Sylow 2-subgroup). Eventually, in 2012, Isaacs and Navarro provided rational groups of order $2^{9} \cdot 3$ with Sylow 2-subgroups that are not rational [27]. However, they also proved the conjecture for solvable groups with Sylow 2-subgroups of nilpotency class at most 2 (see [27, Theorem A]).

Recall that every non-trivial cut group has order divisible by 2 or 3 (see [8, Theorem 1]), and one might wonder which properties are determined locally (i.e. in $p$-subgroups or their normalizers). It is not hard to find cut groups that have Sylow 2-subgroups that are not cut, e.g. the groups with SmallGroupIDs [384, 18033] and $[384,18040]$ of order $2^{7} \cdot 3$. However, for the prime 3 , things seem to behave differently. The following lemma shows that being inverse semi-rational for 3-elements is indeed a somehow local property.

Lemma 6.1. Let $G$ be a group, and let $x \in G$ be a 3-element. Then $x$ is inverse semi-rational in $G$ if and only if $x$ is inverse semi-rational in $P$ for some $P \in \operatorname{Syl}_{3}(G)$.

Proof. Suppose $y$ is an element of a group $Y$. Then $B_{Y}(y):=\mathrm{N}_{Y}(\langle y\rangle) / \mathrm{C}_{Y}(y)$ can naturally be identified with a subgroup of $\operatorname{Aut}(\langle y\rangle)$, the automorphism group of $\langle y\rangle$. By [14, Lemma 5], the element $y$ is inverse semi-rational in $Y$ if and only 
if $B_{Y}(y)\langle\tau\rangle=\operatorname{Aut}(\langle y\rangle)$, where $\tau:\langle y\rangle \rightarrow\langle y\rangle\left(w \mapsto w^{-1}\right)$ denotes the inversion automorphism of $\langle y\rangle$. Recall that the automorphism group of the cyclic group $C_{3}{ }^{f}$ of order $3^{f}$ is cyclic $\left(\operatorname{Aut}\left(C_{3} f\right) \simeq C_{2} \times C_{3}{ }^{f-1}\right)$, and 2 is a primitive root modulo $3^{f}$, i.e. 2 generates the unit group of the ring of integers modulo $3^{f}$. Hence, the 3-element $x$ is inverse semi-rational in an ambient group if and only if $x$ is conjugate to $x^{4}$ in that group. Assume that the 3 -element $x \in G$ of order $3^{f}$ is inverse semi-rational in $G$. Then there exists $g \in \mathrm{N}_{G}(\langle x\rangle)$ such that $x^{g}=x^{4}$. Since the automorphism $w \mapsto w^{4}$ of $\langle x\rangle$ is of 3-power order, we may replace $g$ by its 3 -part, if necessary, and assume that $g$ is also a 3-element. Now, since $g$ normalizes $\langle x\rangle$, the subgroup $S=\langle x, g\rangle$ is a 3-subgroup of $G$. By Sylow's theorem, $S$ is contained in a Sylow 3 -subgroup $P$ of $G$, and $x$ is inverse semi-rational in $P$. The other implication is clear.

The above lemma is clearly false for $p$-elements, $p \geqslant 5$.

It may be observed that if $G$ is a cut group and $P \in \operatorname{Syl}_{3}(G)$, then $P$ is cut if and only if, for all $x \in P$ and for all $S \in \operatorname{Syl}_{3}(G)$ containing $x, x$ is inverse semi-rational in $S$. Lemma 6.1 asserts that each 3-element of a cut group is inverse semi-rational in at least one Sylow 3-subgroup. From the next lemma, one can deduce that it is inverse semi-rational in all Sylow 3-subgroups, in certain cases.

Lemma 6.2. Let $G$ be a cut group and $P \in \operatorname{Syl}_{p}(G)$ for some prime $p$. Then $\exp \mathcal{Z}(P) \mid p$ if $p$ is odd, and $\exp \mathcal{Z}(P) \mid 4$ if $p=2$.

Proof. Let $x \in \mathcal{Z}(P)$ be an element of order $p^{f}$. Then $P \leqslant \mathrm{C}_{G}(x)$, and hence $B_{G}(x)=\mathrm{N}_{G}(\langle x\rangle) / \mathrm{C}_{G}(x)$ has an order not divisible by $p$. Note that, in case $p$ is odd, $\operatorname{Aut}(\langle x\rangle) \simeq C_{p^{f-1}} \times C_{p-1}$, and $\operatorname{Aut}(\langle x\rangle) \simeq C_{2^{f-2}} \times C_{2}$ if $p=2$. Further, by $\left[14\right.$, Lemma 5], we have that $\left[\operatorname{Aut}(\langle x\rangle): B_{G}(x)\right] \leqslant 2$, and thus the result follows.

In particular, abelian Sylow $p$-subgroups of cut groups are elementary abelian for odd primes $p$, and abelian Sylow 2-subgroups of cut groups are of exponent dividing 4 .

Let $p$ be a prime. For a group $X$, denote by $\mathrm{O}_{p}(X)$ and $\mathrm{O}_{p^{\prime}}(X)$ the maximal normal $p$-subgroup of $X$ and maximal normal $p^{\prime}$-subgroup of $X$, respectively. For a group $G$, we can define the upper $p$-series by

$$
1 \leqslant \mathrm{O}_{p^{\prime}}(G) \leqslant \mathrm{O}_{p^{\prime}, p}(G) \leqslant \mathrm{O}_{p^{\prime}, p, p^{\prime}}(G) \leqslant \cdots,
$$

where

$$
\begin{aligned}
\mathrm{O}_{p^{\prime}, p}(G) / \mathrm{O}_{p^{\prime}}(G) & =\mathrm{O}_{p}\left(G / \mathrm{O}_{p^{\prime}}(G)\right), \\
\mathrm{O}_{p^{\prime}, p, p^{\prime}}(G) / \mathrm{O}_{p^{\prime}, p}(G) & =\mathrm{O}_{p^{\prime}}\left(G / \mathrm{O}_{p^{\prime}, p}(G)\right),
\end{aligned}
$$


and so on (alternating between $p$ and $p^{\prime}$ ). If this series terminates in $G$, then $G$ is called $p$-solvable. In this case, the $p$-length of $G$ is defined to be the number of occurrences of the symbol $p$ in the subscript of the first group in the upper $p$-series of $G$ that is equal to $G$. We prove the following.

Proposition 6.3. Let $G$ be a 3-solvable cut group of 3-length at most 1, and let $P \in \operatorname{Syl}_{3}(G)$. Then $P$ is cut.

Proof. Since $G$ has 3-length at most 1, $G$ has normal subgroups $1 \leqslant M \leqslant N \leqslant G$ such that $M$ and $G / N$ are $3^{\prime}$-groups and $N / M$ is a 3-group. Let $x \in P$. Then $x$ is inverse semi-rational in $G$ and hence also in some Sylow 3-subgroup $Q$ of $G$ by Lemma 6.1. Since $N$ is a normal subgroup of $3^{\prime}$-index, $Q$ is contained in $N$, and hence $x$ is inverse semi-rational in $N$. Note that $M$ is a normal complement for $P$ in $N$, that is, $N=M P$ is the semi-direct product of the normal subgroup $M$ with $P$, and the restriction to $P$ of the projection map $\pi: N=M P \rightarrow P$ is the identity map on $P$. As $x$ is inverse semi-rational in $N$, there is $n \in N$ such that $x^{n}=x^{4}$. Write $n=m y, m \in M, y \in P$. Then $x^{y}=x^{4}$ since $\pi(n)=y$. Hence, $x$ is inverse semi-rational in $P$, implying that $P$ is cut.

The following proposition follows from Lemma 6.2 and Proposition 6.3.

Proposition 6.4. Let $G$ be a cut group and $P \in \operatorname{Syl}_{3}(G)$. Then $P$ is also cut, provided one of the following holds:

(1) $P$ is abelian,

(2) $P$ is a normal subgroup of $G$.

Remark 6.5. Since the examples of Isaacs and Navarro of rational groups with non-rational Sylow 2-subgroups are of order $2^{9} \cdot 3$, one might be tempted to think that cut groups of order $2 \cdot 3^{a}$ could serve to find examples of cut groups whose Sylow 3-subgroups are not cut. However, this is not the case. Indeed, by Cayley's normal 2-complement theorem together with the previous proposition, we obtain that if $G$ is a cut group with order only divisible by the primes 2 and 3 and with cyclic Sylow 2-subgroups (for example, $|G|=2 \cdot 3^{a}$ ), then any Sylow 3-subgroup of $G$ is cut.

Theorem 6.6. Let $G$ be a cut group and $P \in \mathrm{Syl}_{3}(G)$. Then $P$ is also cut, provided one of the following holds:

(1) $G$ is supersolvable,

(2) $G$ is a Frobenius group, 
(3) $G$ is simple,

(4) $G$ is of odd order and $\mathrm{O}_{3}(G)$ is abelian.

Proof. (1) Supersolvable groups have a Sylow tower [23, VI, Satz 9.1], so in particular $p$-length at most 1 for all primes $p$. The claim thus follows from Proposition 6.3.

(2) Assume that $G$ is a Frobenius group with Frobenius kernel $F$ and Frobenius complement $K$. Since $F$ and $K$ have coprime order, a Sylow 3-subgroup $P$ of $G$ is isomorphic to a Sylow 3-subgroup of $F$ or to a Sylow 3-subgroup of $K$. Assume that 3 divides $|F|$. Since $F$ is nilpotent and characteristic in $G, P$ is normal in $G$. Hence, it is cut by Proposition 6.4. If 3 divides $|K|$, then $P$ is cyclic by [28, Theorem 11.4.5(4)], and it follows from Lemma 6.2 that it is also cut. (Of course, we could also have used the description of Frobenius cut groups in [3, Theorem 1.3].)

(3) In Theorem B, the simple cut groups were determined. They are given along with the nilpotency class of their Sylow 3-subgroup $P$ in Table 1, showing that there nilpotency class can exceed 2. Using for example GAP, one can check that these Sylow 3 -subgroups are again cut.

(4) In the case a group has odd order, then inverse semi-rational is the same as semi-rational by [14, Remark 13]. These groups have been described in [14, Theorem 3]. They come in three families: 3-groups, certain Frobenius groups and certain groups that are occasionally called 2-Frobenius or double Frobenius groups. Only in the last case, we still need to verify the claim. By [14, Theorem 3 (2)], they have the following structure: $|G|=7 \cdot 3^{b}$, and $G$ contains a normal Frobenius subgroup of index 3 with $\mathrm{O}_{3}(G)$ the Frobenius kernel. Moreover, $\mathrm{O}_{3}(G) T \in \operatorname{Syl}_{3}(G)$ for some subgroup $T=\langle t\rangle$ of order 3 , and $G / \mathrm{O}_{3}(G)$ is the non-abelian group of order 21.

Assume that $\mathrm{O}_{3}(G)$ is abelian. We aim to show that every element of the Sylow 3-subgroup $P=\mathrm{O}_{3}(G)\langle t\rangle$ is inverse semi-rational in $P$. Firstly, we claim that, in this setting, $\mathrm{O}_{3}(G)$ has exponent 3. Assume there is $y \in \mathrm{C}_{\mathrm{O}_{3}(G)}(t)$ of order 9.

\begin{tabular}{lrrrrrrrrr}
\hline$G$ & $C_{2}$ & $C_{3}$ & $A_{7}$ & $A_{8}$ & $A_{9}$ & $A_{12}$ & $L_{2}(7)$ & $U_{3}(3)$ & $U_{3}(5)$ \\
$\operatorname{cl}(P)$ & 0 & 1 & 1 & 1 & 3 & 3 & 1 & 2 & 1 \\
\hline$G$ & $U_{4}(3)$ & $U_{5}(2)$ & $U_{6}(2)$ & $S_{4}(3)$ & $S_{6}(2)$ & $O_{8}^{+}(2)$ & $M_{11}$ & $M_{12}$ & $M_{22}$ \\
$\operatorname{cl}(P)$ & 3 & 3 & 3 & 3 & 3 & 3 & 1 & 2 & 1 \\
\hline$G$ & $M_{23}$ & $M_{24}$ & $C o_{1}$ & $C o_{2}$ & $C o_{3}$ & $H S$ & $M c L$ & $T h$ & $M$ \\
$\operatorname{cl}(P)$ & 1 & 2 & 5 & 3 & 3 & 1 & 3 & 7 & 9 \\
\hline
\end{tabular}

Table 1. Simple cut groups $G$ with the nilpotency class of their Sylow 3-subgroup $P$ 
Then $[t, y]=1$, and hence $t \in \mathrm{C}_{G}(y)$. However, $\mathrm{C}_{G}(y)=\mathrm{O}_{3}(G)$ : clearly, we have $\mathrm{O}_{3}(G) \leqslant \mathrm{C}_{G}(y)$; since the former is abelian, but also since $\mathrm{O}_{3}(G) F$ is a Frobenius group for every $F \in \operatorname{Syl}_{7}(G), \mathrm{C}_{G}(y)$ cannot contain elements of order 7, and since $y$ is inverse semi-rational in $G,\left[\mathrm{~N}_{G}(\langle y\rangle): \mathrm{C}_{G}(y)\right]=3$, so indeed, $\mathrm{C}_{G}(y)=\mathrm{O}_{3}(G)$. Summing up, $t \in \mathrm{C}_{G}(y)=\mathrm{O}_{3}(G)$. This yields a contradiction since $t \notin \mathrm{O}_{3}(G)$ by the form of the Sylow 3-subgroup. So there is no element of order 9 in $\mathrm{C}_{\mathrm{O}_{3}(G)}(t)$ and $\exp \left(\mathrm{C}_{\mathrm{O}_{3}(G)}(t)\right)=3$. By [29, Lemma 2.4], $\mathrm{O}_{3}(G)=\left\langle\mathrm{C}_{\mathrm{O}_{3}(G)}(t)^{f} \mid f \in F\right\rangle$ for some $F \in \operatorname{Syl}_{7}(G)$, so also $\exp \left(\mathrm{O}_{3}(G)\right)=3$ since the latter is abelian. Thus, every element of $\mathrm{O}_{3}(G)$ is inverse semi-rational in $\mathrm{O}_{3}(G)$ and hence also in $P \geqslant \mathrm{O}_{3}(G)$.

Now assume that $x \in P \backslash \mathrm{O}_{3}(G)$. Then $P$ is the unique Sylow 3-subgroup of $G$ containing $x$ because $\mathrm{O}_{3}(G)<\left\langle\mathrm{O}_{3}(G), x\right\rangle \leqslant P$ and $\left[P: \mathrm{O}_{3}(G)\right]=3$. Since $x$ is inverse semi-rational in $G$, Lemma 6.1 asserts that $x$ is inverse semi-rational in a Sylow 3-subgroup that contains it. Hence, $x$ is inverse semi-rational in $P$. Consequently, every element of $P$ is inverse semi-rational in $P$, i.e. $P$ is cut.

Recently, N. Grittini proved that the assumption that $\mathrm{O}_{3}(G)$ is abelian in (4) is superfluous: the Sylow 3-subgroups of cut groups of odd order are always cut [20].

Note that the class of groups in (4) contains groups of 3-length 2. Theorem 6.6 could also have been proved using the dual characterization of cut groups using characters.

Recall that a group $G$ is called a Camina group if $G^{\prime} \neq G$ and, for every $g \notin G^{\prime}$, the coset $g G^{\prime}$ is a conjugacy class. As Camina groups are either Frobenius or p-groups [16], we immediately have the following corollary from Theorem 6.6.

Corollary 6.7. A Sylow 3-subgroup of a Camina cut group is again a cut group.

Using the above results and GAP with the grpconst package [10], we have verified that the Sylow 3-subgroups of all cut groups of order at most 2000, as well as for order $2^{2} \cdot 3^{6}, 2^{3} \cdot 3^{6}$ and $2^{2} \cdot 3^{7}$, are again cut. Naturally, the following question arises.

Question 6.8. Are Sylow 3-subgroups of cut groups again cut?

Question 6.8 essentially asks whether we can always replace the existence assertion for the Sylow 3-subgroup in Lemma 6.1 by a "for all" statement.

Remark 6.9. Recall that (normal) subgroups of cut groups need not be cut groups. But for classes for which Question 6.8 has a positive answer, the Sylow 3-subgroups have this property. In view of Proposition 2.1, this implies that, for such groups, the centers of the Wedderburn components of the rational group algebra of cut groups also influence those of its Sylow 3-subgroups. For, if the centers of 
the Wedderburn components of $\mathbb{Q} G$ are all rational or quadratic imaginary, then the same holds true for $\mathbb{Q} P$ for $P \in \operatorname{Syl}_{3}(G)$.

\section{Existence of cut groups}

In this section, we will give some indications that the class of cut groups is surprisingly large in all finite groups. Recall that a $p$-group can only be a cut group if $p \in\{2,3\}$ (see [8, Theorem 1]). We show that, in these cases, the ratio of cut groups tends to one in the logarithmic sense.

Proposition 7.1. Let $c(r)$ denote the number of cut groups of order $r$ and $f(r)$ the number of all groups of order $r$. Then

$$
\lim _{n \rightarrow \infty} \frac{\ln c\left(p^{n}\right)}{\ln f\left(p^{n}\right)}=1 \quad \text { for } p \in\{2,3\} .
$$

Proof. In their work on asymptotic behavior of the number of $p$-groups, Higman, Sims, Newman and Seeley showed that, for each prime $p$,

$$
p^{\frac{2}{27} m^{2}(m-6)} \leqslant f\left(p^{m}\right) \leqslant p^{\frac{2}{27} m^{3}+O\left(m^{\frac{5}{2}}\right)} ;
$$

see [11, Theorem 4.5, Theorem 5.7]. Noting that all the groups constructed by Higman to obtain the lower bound are actually cut groups in the case $p=2$ (as they are extensions of elementary abelian 2-groups by elementary abelian 2 -groups; see [11, Section 4]) shows that $(* *)$ holds for $p=2$. By [11, Theorem 19.3], it follows that the number of 3-groups of exponent 3 (and nilpotency class 2 ) has the same asymptotic behavior in the leading term, so (**) also holds for $p=3$.

Remark 7.2. Though the above proposition shows that there are many cut groups in the class of $p$-groups, it says nothing about the actual percentage. For groups of small order, we now also support this with numerical data which we obtained using GAP and the SgIPPow package [17]. Table 2 lists the number and percentage of rational as well as cut 2-groups up to order $2^{9}$. Observe that, for the 2-groups, the percentage of cut groups increases again from order $2^{8}$. The number and percentage of cut 3 -groups up to order $3^{8}$ are listed in Table 3 (note that the only rational group of odd order is the trivial one). Also, in Table 4, we list the number and percentage of rational and cut groups of selected mixed orders. Figure 1 presents a chart showing the percentage of groups that are rational and cut, up to order 1023. The horizontal and the vertical axes indicate the order and the percentage, respectively. The dashed graph gives the percentage of rational groups up to that order, 


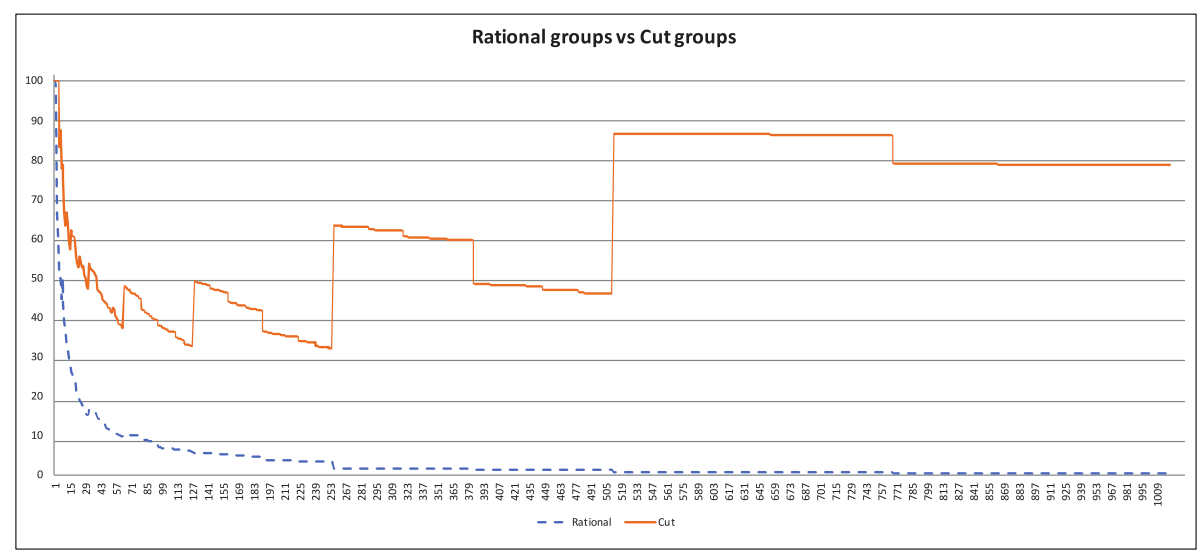

Figure 1. Rational and cut groups of order at most 1023

\begin{tabular}{|c|c|c|c|c|c|}
\hline \multirow[t]{2}{*}{ Order } & \multirow{2}{*}{$\begin{array}{l}\text { Number of } \\
\text { all groups }\end{array}$} & \multicolumn{2}{|c|}{ Rational groups } & \multicolumn{2}{|c|}{ cut groups } \\
\hline & & Number & Percentage & Number & Percentage \\
\hline $2^{2}=4$ & 2 & 1 & $50 \%$ & 2 & $100 \%$ \\
\hline $2^{3}=8$ & 5 & 3 & $60 \%$ & 4 & $80 \%$ \\
\hline $2^{4}=16$ & 14 & 3 & $21.43 \%$ & 10 & $71.43 \%$ \\
\hline $2^{5}=32$ & 51 & 10 & $19.61 \%$ & 33 & $64.71 \%$ \\
\hline $2^{6}=64$ & 267 & 30 & $11.24 \%$ & 161 & $60.30 \%$ \\
\hline $2^{7}=128$ & 2328 & 124 & $5.33 \%$ & 1349 & $57.95 \%$ \\
\hline $2^{8}=256$ & 56092 & 748 & $1.34 \%$ & 37593 & $67.02 \%$ \\
\hline $2^{9}=512$ & 10494213 & 59514 & $0.57 \%$ & 9127858 & $86.98 \%$ \\
\hline
\end{tabular}

Table 2. Rational and cut groups in small order 2-groups

whereas the solid graph indicates the percentages of cut groups up to that order. Note that the upward bumps for the percentage of cut groups appear at 2-powers, whereas the (visible) bumps downwards for this percentage happen at orders of the form $2^{a} \cdot 3$. Yet, also for these orders, the cut groups are still surprisingly numerous; cf. Table 4. Also, when considering all groups up to a certain small order, cut groups are not negligible. For instance, about $86.62 \%$ of the groups of order at most 512 and $78.55 \%$ of groups of order at most 1023 are cut groups, whereas $0.57 \%$ of the groups of order at most 512 and $0.52 \%$ of groups of order at most 1023 are rational. 


\begin{tabular}{lrrr}
\hline Order & $\begin{array}{r}\text { Number of } \\
\text { all groups }\end{array}$ & \multicolumn{2}{c}{ cut groups } \\
\cline { 3 - 4 } & & Number & Percentage \\
\hline $3^{2}=9$ & 2 & 1 & $50 \%$ \\
$3^{3}=27$ & 5 & 3 & $60 \%$ \\
$3^{4}=81$ & 15 & 4 & $26.67 \%$ \\
$3^{5}=243$ & 67 & 14 & $20.90 \%$ \\
$3^{6}=729$ & 504 & 96 & $19.05 \%$ \\
$3^{7}=2187$ & 9310 & 595 & $6.39 \%$ \\
$3^{8}=6561$ & 1396077 & 66312 & $5.06 \%$ \\
\hline
\end{tabular}

Table 3. cut groups in small order 3-groups

\begin{tabular}{lrrrrrr}
\hline \multirow{2}{*}{ Order } & $\begin{array}{r}\text { Number of } \\
\text { all groups }\end{array}$ & \multicolumn{2}{c}{ Rational groups } & & \multicolumn{2}{c}{ cut groups } \\
\cline { 7 - 7 } \cline { 6 - 7 } & & Number & Percentage & & Number & Percentage \\
\hline $2^{2} \cdot 5=20$ & 5 & 0 & $0 \%$ & 1 & $20 \%$ \\
$2 \cdot 3 \cdot 7=42$ & 6 & 0 & $0 \%$ & 2 & $33.33 \%$ \\
$2^{6} \cdot 3=192$ & 1543 & 18 & $1.17 \%$ & 318 & $20.61 \%$ \\
$2^{7} \cdot 3=384$ & 20169 & 317 & $0.32 \%$ & & 2279 & $11.30 \%$ \\
$2^{3} \cdot 5^{2}=400$ & 221 & 1 & $0.45 \%$ & 12 & $5.43 \%$ \\
$2^{6} \cdot 3^{2}=576$ & 8681 & 45 & $0.52 \%$ & & 1074 & $12.37 \%$ \\
$2^{3} \cdot 3 \cdot 7^{2}=588$ & 66 & 0 & $0 \%$ & 4 & $6.06 \%$ \\
$2^{3} \cdot 3^{4}=648$ & 757 & 11 & $1.45 \%$ & 151 & $19.95 \%$ \\
$2^{8} \cdot 3=768$ & 1090235 & 304 & $0.03 \%$ & 64765 & $5.94 \%$ \\
$2 \cdot 3^{6}=1458$ & 1798 & 1 & $0.06 \%$ & 387 & $21.52 \%$ \\
$2^{3} \cdot 3^{2} \cdot 5^{2}=1800$ & 749 & 0 & $0 \%$ & 14 & $1.87 \%$ \\
$2^{3} \cdot 3^{5}=1944$ & 3973 & 17 & $0.43 \%$ & 525 & $13.21 \%$ \\
\hline
\end{tabular}

Table 4. Rational and cut groups for some selected mixed orders

Question 7.3. Let $f$ and $c$ be defined as in Proposition 7.1. Is there $m \in \mathbb{Z} \geqslant 1$ such that

$$
\frac{c\left(3^{m+1}\right) f\left(3^{m}\right)}{c\left(3^{m}\right) f\left(3^{m+1}\right)}>1,
$$

i.e. does the proportion of the cut 3 -groups in all 3-groups increase again at a certain point (as it does for 2-groups at $2^{8}$ )? 
Acknowledgments. We are grateful to Urban Jezernik, Gabriel Navarro, Inder Bir Singh Passi and Ángel del Río for very interesting discussions. We want to thank Jürgen Müller for providing us with the Sylow 3-subgroups of some sporadic simple groups. We are grateful to Benjamin Sambale for the proof of the improved Theorem A. The fourth author is thankful to Eric Jespers and FWO for the local hospitality provided for stay at Vrije Universiteit Brussel, which played a vital role in the outcome of this paper. We want to thank Adalbert and Victor Bovdi for pointing out the existence of the articles $[12,37]$ to us. We wish to thank the reviewers for suggesting changes that improved the readability of the paper.

\section{Bibliography}

[1] S. H. Alavi and A. Daneshkhah, On semi-rational finite simple groups, Monatsh. Math. 184 (2017), no. 2, 175-184.

[2] R. Z. Aleev, A. V. Kargapolov and V. V. Sokolov, The ranks of central unit groups of integral group rings of alternating groups, Fundam. Prikl. Mat. 14 (2008), no. 7, 15-21.

[3] A. Bächle, Integral group rings of solvable groups with trivial central units, Forum Math. 30 (2018), no. 4, 845-855.

[4] A. Bächle, 3 questions on cut groups, Adv. Group Theory Appl. 8 (2019), 157-160.

[5] A. Bächle, G. Janssens, E. Jespers, A. Kiefer and D. Temmerman, Abelianization and fixed point properties of units in integral group rings, preprint (2018), https:// arxiv.org/abs/1811.12184; to appear in Math. Nachr.

[6] A. Bächle, G. Janssens, E. Jespers, A. Kiefer and D. Temmerman, A dichotomy for integral group rings via higher modular groups as amalgamated products, preprint (2018), https://arxiv.org/abs/1811.12226.

[7] A. Bächle, S. Maheshwary and L. Margolis, Abelianization of the unit group of an integral group ring, preprint (2020), https://arxiv.org/abs/2004.03173; to appear in Pacific J. Math.

[8] G. K. Bakshi, S. Maheshwary and I. B. S. Passi, Integral group rings with all central units trivial, J. Pure Appl. Algebra 221 (2017), no. 8, 1955-1965.

[9] G. K. Bakshi, S. Maheshwary and I. B.S. Passi, Group rings and the RS property, Comm. Algebra 47 (2019), no. 3, 969-977.

[10] H. U. Besche and B. Eick, Grpconst, https://www.gap-system.org/Packages/grp const.html, 2018, GAP package, Version 2.6.

[11] S. R. Blackburn, P. M. Neumann and G. Venkataraman, Enumeration of Finite Groups, Cambridge Tracts in Math. 173, Cambridge University, Cambridge, 2007. 
[12] A. A. Bovdi, The multiplicative group of an integral group ring (Mul'tiplikativnaya gruppa tselochislennogo gruppovogo kol'tsa), Kod GASNTI 27.17.19, Uzhgorodskij Gosudarstvennyj Universitet, Uzhgorod, 1987.

[13] A. M. Broshi, Galois correspondences between the irreducible characters and the conjugacy classes of finite groups, J. Algebra 19 (1971), 441-451.

[14] D. Chillag and S. Dolfi, Semi-rational solvable groups, J. Group Theory 13 (2010), no. $4,535-548$.

[15] J. H. Conway, R. T. Curtis, S. P. Norton, R. A. Parker and R. A. Wilson, Atlas of Finite Groups, Oxford University, Eynsham, 1985.

[16] R. Dark and C. M. Scoppola, On Camina groups of prime power order, J. Algebra 181 (1996), no. 3, 787-802.

[17] B. Eick and M. Vaughan-Lee, SglPPow, https://gap-packages.github.io/sglppow/, 2014, GAP package, Version 1.1.

[18] R. A. Ferraz, Simple components and central units in group algebras, J. Algebra 279 (2004), no. 1, 191-203.

[19] R. Gow, Groups whose characters are rational-valued, J. Algebra 40 (1976), no. 1, 280-299.

[20] N. Grittini, A note on cut groups of odd order, preprint (2020), https://arxiv.org/abs/ 1911.13196v4.

[21] P. Hegedús, Structure of solvable rational groups, Proc. Lond. Math. Soc. (3) 90 (2005), no. 2, 439-471.

[22] G. Higman, The units of group-rings, Proc. Lond. Math. Soc. (2) 46 (1940), 231-248.

[23] B. Huppert, Endliche Gruppen. I, Grundlehren Math. Wiss. 134, Springer, Berlin, 1967.

[24] I. M. Isaacs, Character Theory of Finite Groups, AMS Chelsea, Providence, 2006.

[25] I. M. Isaacs and D. Karagueuzian, Conjugacy in groups of upper triangular matrices, J. Algebra 202 (1998), no. 2, 704-711.

[26] I. M. Isaacs and D. Karagueuzian, Erratum: "Conjugacy in groups of upper triangular matrices" [J. Algebra 202 (1998), no. 2, 704-711; MR1617655 (99b:20011)], J. Algebra 208 (1998), no. 2, 722.

[27] I. M. Isaacs and G. Navarro, Sylow 2-subgroups of rational solvable groups, Math. Z. 272 (2012), no. 3-4, 937-945.

[28] E. Jespers and A. del Río, Group Ring Groups. Vol. 1. Orders and Generic Constructions of Units, De Gruyter, Berlin, 2015.

[29] E. Khukhro, N. Makarenko and P. Shumyatsky, Frobenius groups of automorphisms and their fixed points, Forum Math. 26 (2014), no. 1, 73-112. 
[30] D. Kletzing, Structure and Representations of Q-Groups, Lecture Notes in Math. 1084, Springer, Berlin, 1984.

[31] S. Maheshwary, Integral group rings with all central units trivial: Solvable groups, Indian J. Pure Appl. Math. 49 (2018), no. 1, 169-175.

[32] S. Maheshwary and I. B. S. Passi, The upper central series of the unit groups of integral group rings: a survey, in: Group theory and Computation, Indian Stat. Inst. Ser., Springer, Singapore (2018), 175-195.

[33] E. Marberg, Exotic characters of unitriangular matrix groups, J. Pure Appl. Algebra 216 (2012), no. 2, 239-254.

[34] G. Navarro, Problems in character theory, in: Character Theory of Finite Groups, Contemp. Math. 524, American Mathematical Society, Providence (2010), 97-125.

[35] G. Navarro and J. Tent, Rationality and Sylow 2-subgroups, Proc. Edinb. Math. Soc. (2) 53 (2010), no. 3, 787-798.

[36] G. Navarro and P. H. Tiep, Rational irreducible characters and rational conjugacy classes in finite groups, Trans. Amer. Math. Soc. 360 (2008), no. 5, 2443-2465.

[37] Z. F. Pataŭ, The multiplicative group of a group ring, in: Studies in The Qualitative Theory of Differential Equations and Its Applications (in Ukrainian), Akad. Nauk Ukrain. SSR Inst. Mat., Kiev (1978), 47-48, 75.

[38] J. Ritter and S. K. Sehgal, Integral group rings with trivial central units, Proc. Amer. Math. Soc. 108 (1990), no. 2, 327-329.

[39] G. R. Robinson and J. G. Thompson, Sums of squares and the fields $\mathbf{Q}_{A_{n}}$, J. Algebra 174 (1995), no. 1, 225-228.

[40] J. J. Rotman, An Introduction to the Theory of Groups, 4th ed., Grad. Texts in Math. 148, Springer, New York, 1995.

[41] S. K. Sehgal, Units in Integral Group Rings, Pitman Monogr. Surv. Pure Appl. Math. 69, Longman Scientific \& Technical, Harlow, 1993.

[42] J. F. Tent, Quadratic rational solvable groups, J. Algebra 363 (2012), 73-82.

[43] The GAP Group, GAP - Groups, Algorithms, and Programming, Version 4.9.1, 2018, https://www.gap-system.org.

Received October 15, 2020; revised April 27, 2021.

\section{Author information}

Andreas Bächle, Department of Mathematics and Data Science,

Vrije Universiteit Brussel, Pleinlaan 2, 1050 Brussels, Belgium.

E-mail: abaechle@gmx.net 
Mauricio Caicedo, Department of Mathematics and Data Science, Vrije Universiteit Brussel, Pleinlaan 2, 1050 Brussels, Belgium.

E-mail: mcaicedo@vub.be

Corresponding author:

Eric Jespers, Department of Mathematics and Data Science, Vrije Universiteit Brussel, Pleinlaan 2, 1050 Brussels, Belgium. E-mail: eric.jespers@vub.be

Sugandha Maheshwary, Department of Mathematical Sciences, Indian Institute of Science Education and Research Mohali, Sector 81, Mohali (Punjab)-140306, India.

E-mail: sugandha@iisermohali.ac.in 